7th International Workshop on Astronomy and

Relativistic Astrophysics (IWARA 2016)

International Journal of Modern Physics: Conference Series

Vol. 45 (2017) 1760068 (5 pages)

(C) The Author(s)

DOI: $10.1142 / \mathrm{S} 2010194517600680$

\title{
Photon-Photon Interaction in Glueball Production
}

\author{
Daniel T. da Silva, Mario L. L. da Silva \\ Instituto de Física e Matemática \\ Universidade Federal de Pelotas (UFPel) \\ Rua dos Ipês, Capão do Leão, 96050-500 Pelotas, RS, Brazil \\ neodts@gmail.com, mllsilva@gmail.com \\ Dimiter Hadjimichef \\ Instituto de Física \\ Universidade Federal do Rio Grande do Sul (UFRGS) \\ Av. Bento Gonçalves 9500, Agronomia, 91501-970 Porto Alegre, RS, Brazil \\ dimihdj@gmail.com
}

Published 15 August 2017

\begin{abstract}
In the last years many exotic states have been identified in several colliders around the world. One of the exotic states provided in QCD is the glueball. Using a non-relativistic gluon bound-state model, we compute $\Gamma(G \rightarrow \gamma \gamma)$, where $G$ is a pseudoscalar, tensor, or scalar digluon. For the starting from the amplitudes we considers the process $\gamma \gamma \rightarrow g^{*} g^{*}$ at threshold, where the amplitudes are obtained in perturbative QCD at lowest order by deriving them from QED calculation and the $g^{*} \mathrm{~s}$ are massive constituent gluons. In this calculation the unknown parameters of the model, such as the digluon wave function, are obtained using measured values of $\Gamma(J / \Psi \rightarrow G \gamma)$. Our theorical results are compared with the present experimental limits for the various glueballs candidates.
\end{abstract}

Keywords: Meson Production, Meson Decay, Glueball.

PACS numbers:12.39.Mk; 12.38.-t; 14.40.-n

\section{Introduction}

The self-interaction of gluons opens the possibility for the existence of glueballs, bound states of gluons. These states are predicted by several phenomenological models and lattice calculations which have been used to describe their proprieties (for a review see Ref. ${ }^{1}$ ). The hadrons proprieties can help us to determine their structure and on this way we need precise measurements of these proprieties to compare the results obtained with phenomenological and theoretical models. An interesting way

This is an Open Access article published by World Scientific Publishing Company. It is distributed under the terms of the Creative Commons Attribution 4.0 (CC-BY) License. Further distribution of this work is permitted, provided the original work is properly cited. 
to investigate the hadrons internal structure is through the decay width of hadrons in particular the $\gamma \gamma$ channel is interesting when we consider glueballs.

In this work we shall estimate the decay rates of some glueball candidates in two photons and to compare our results with experimental data. The decay rates will be obtained in the context of a non-relativistic gluon bound state model (Ref. ${ }^{2}$ ).

\section{Formalism}

Here we shall consider the formalism used by Kada et al (Ref. ${ }^{2}$ ) to estimate the glueball decay into photon-photon. This model is based on the two-gluon BetheSalpeter wave function in its non-relativistic form. On this way one can neglect the components of the relative four-momenta between the gluons because they are to small compared to the glueball mass $M_{G}$ if one goes to the rest frame. The formalism relate the glueball decay $G \rightarrow \gamma \gamma$ to the $g^{*} g^{*} \rightarrow \gamma \gamma$ interaction, where $g *$ is related to the massive gluon and the gluon mass is about $M_{G} / 2$. The authors in Ref. ${ }^{3}$ have reported some misprints in a few expressions in this calculation which we will correct here. The decay amplitude for this process is given in terms of the helicity amplitudes by the following expression

$$
\begin{aligned}
\mathcal{M}_{G \rightarrow \gamma \gamma}^{\lambda_{\gamma} \lambda_{\gamma}^{\prime}}= & (-i)^{L}\left(\frac{2}{M_{G}}\right)^{L+1 / 2} \frac{(2 L+1) ! !}{L !}\left[\left(\frac{d}{d r}\right)^{L} R_{L}(r)\right]_{r=0} \\
& \times \lim _{\beta \rightarrow 0} \frac{1}{(\beta)^{L}} \int \frac{d(\cos \theta)}{2} \sum_{\lambda \lambda^{\prime}} \chi_{\lambda \lambda^{\prime}}^{L S J \Lambda}(\theta) \mathcal{M}_{g^{*} g^{*} \rightarrow \gamma \gamma}^{\lambda_{\gamma} \lambda_{\gamma}^{\prime}, \lambda \lambda^{\prime}}(\theta),
\end{aligned}
$$

where $\lambda_{\gamma}, \lambda_{\gamma}^{\prime}, \lambda, \lambda^{\prime}$ are the helicities of the photons and gluons, respectively and by angular momentum conservation $\lambda_{\gamma}-\lambda_{\gamma}^{\prime}=\Lambda ; R_{L}(r)$ is the radial part of the wave function of the two-gluon system; $\theta$ is the scattering angle in the center of mass frame and $\beta$ is the gluon velocity; the function $\chi_{\lambda \lambda^{\prime}}^{L S J}(\theta)$ gives the projection of the $\lambda, \lambda^{\prime}$ onto L,S,J, $\Lambda$ state of the glueball gives in terms of Clebsch-Gordan coefficients and the Wigner rotation matrix element

$$
\chi_{\lambda \lambda^{\prime}}^{L S J \Lambda}(\theta)=\left(\frac{2 L+1}{4 \pi}\right)^{1 / 2}\left\langle 11 \lambda-\lambda^{\prime} \mid 11 S \bar{\Lambda}\right\rangle\langle L S 0 \bar{\Lambda} \mid L S J \bar{\Lambda}\rangle d_{\Lambda \bar{\Lambda}}^{J}(\theta)
$$

with $\bar{\Lambda}=\lambda-\lambda^{\prime}$.

The results for the decay widths are still not yet accessible because we do not know some parameters such as $R_{0}(0)$. The solution of this matter is normalize the results to eliminate the unknown parameters (Ref. ${ }^{2}$ ). The correction of the amplitudes can be fixed using the process $J / \psi \rightarrow \gamma G$. The expressions used to describe this process is similar to Eq. (1), then we obtain the ratio $\Gamma(G \rightarrow \gamma \gamma) / \Gamma(J / \psi \rightarrow \gamma G)$ and so we have no more adjustable parameter. 


\section{Glueball $0^{++}$}

The decay amplitude for the scalar glueball $(J=L=S=\Lambda=0)$ is given by

$$
\begin{aligned}
\mathcal{M}_{G \rightarrow \gamma \gamma}^{\lambda_{\gamma} \lambda_{\gamma}^{\prime}}= & \frac{1}{M_{G}^{1 / 2} \sqrt{6 \pi}} R_{0}(0) \lim _{\beta \rightarrow 0} \frac{1}{\beta} \int \frac{d(\cos \theta)}{2} \\
& \times\left(\mathcal{M}^{\lambda_{\gamma} \lambda_{\gamma}^{\prime},++}+\mathcal{M}^{\lambda_{\gamma} \lambda_{\gamma}^{\prime},--}-\mathcal{M}^{\lambda_{\gamma} \lambda_{\gamma}^{\prime}, 00}\right) .
\end{aligned}
$$

In this case the photon helicities are ++ and -- than the helicity amplitudes obtained in the Appendix A of Ref. ${ }^{2}$ are

$$
\begin{aligned}
\mathcal{M}^{++, \pm \pm} & =-3 C\left[1+\cos ^{2} \theta \mp 2 \beta \sin \theta+\beta^{2}\left(-2+7 \cos ^{2} \theta \sin ^{2} \theta\right)\right], \\
\mathcal{M}^{++, 00} & =6 C \sin ^{2} \theta\left(1-7 \beta^{2} \cos ^{2} \theta\right),
\end{aligned}
$$

where $\theta$ is the center of mass scattering angle and

$$
C=\left(8 \sqrt{2} \alpha \alpha_{s}\right) / 9 \text {. }
$$

The remaining helicity amplitudes for the scalar glueball are given by

$$
\mathcal{M}^{-\lambda_{\gamma}-\lambda_{\gamma}^{\prime}-\lambda-\lambda^{\prime}}=(-)^{\lambda+\lambda^{\prime}} \mathcal{M}^{\lambda_{\gamma} \lambda_{\gamma}^{\prime} \lambda \lambda^{\prime}} .
$$

Therefore the amplitude for the decay of scalar glueball is

$$
\mathcal{M}_{G\left(0^{++}\right) \rightarrow \gamma \gamma}^{\lambda_{\gamma} \lambda_{\gamma}^{\prime}}=\frac{32 \alpha \alpha_{s}}{3 M_{G}^{1 / 2} \sqrt{3 \pi}} R_{0}(0)
$$

and the decay width is

$$
\Gamma\left(G\left(0^{++}\right) \rightarrow \gamma \gamma\right)=\frac{64 \alpha^{2} \alpha_{s}^{2}}{27 M_{G}^{2} \pi^{2}}\left|R_{0}(0)\right|^{2} .
$$

To eliminate the unknown parameters we must use the $J / \psi$ radiative decay width to $\gamma G$ which can be calculated using Eq. (3) and the following helicity amplitudes extracted from Appendix B of Ref. ${ }^{2}$

$$
\begin{gathered}
\mathcal{M}^{++, \pm \pm}=-K_{\theta}\left[\left(1+\cos ^{2} \theta\right)\left(1-\beta^{2}\right) \mp 2 y \beta(1 \mp \beta)^{2}\right] \\
\mathcal{M}^{++, 00}=2 K_{\theta} \sin ^{2} \theta\left(1-\beta^{2}\right),
\end{gathered}
$$

where

$$
K_{\theta}=\frac{128 \pi^{3 / 2} \sqrt{\alpha} \alpha_{s} f_{\psi}}{9 M_{\psi}\left[\left(1+y \beta^{2}\right)^{2}-(1-y)^{2} \beta^{2} \cos ^{2} \theta\right]},
$$

with $y=M_{G}^{2} / M_{\psi}^{2}$ and $f_{\psi} \simeq 0.27 \mathrm{GeV}$. The remaining helicity amplitude for this case can be obtained by

$$
\mathcal{M}^{-\lambda_{\psi}-\lambda_{\gamma}-\lambda-\lambda^{\prime}}=-(-)^{\lambda_{\psi}+\lambda+\lambda^{\prime}} \mathcal{M}^{\lambda_{\psi} \lambda_{\gamma} \lambda \lambda^{\prime}}
$$


Therefore the amplitude for the radiative decay $J / \psi$ to scalar glueball is

$$
\mathcal{M}_{J / \psi \rightarrow \gamma G\left(0^{++}\right)}^{\lambda_{\gamma} \lambda_{\gamma}^{\prime}}=\frac{128 \sqrt{2} \pi \sqrt{\alpha} \alpha_{s} f_{\psi}}{9 \sqrt{3} M_{G}^{1 / 2} M_{\psi}} R_{0}(0),
$$

and the decay width is

$$
\Gamma(J / \psi \rightarrow \gamma G)=\frac{4096 \pi \alpha \alpha_{s}^{2} f_{\psi}^{2}}{729 M_{G} M_{\psi}^{3}}\left(1-\frac{M_{G}^{2}}{M_{\psi}^{2}}\right)\left|R_{0}(0)\right|^{2} .
$$

The normalized decay width is

$$
\frac{\Gamma\left(G_{0^{++}} \rightarrow \gamma \gamma\right)}{\Gamma\left(J / \Psi \rightarrow \gamma G_{0^{++}}\right)}=\frac{27 \alpha}{64 \pi^{3}} \frac{M_{\Psi}^{3}}{f_{\Psi}^{2} M_{G}} \frac{1}{\left(1-M_{G}^{2} / M_{\Psi}^{2}\right)} .
$$

\section{Results}

Numerical results for the decay width of glueballs to $\gamma \gamma$ can be obtained from Eq. (16).This result is obtained in terms of experimental data for the radiative decay of $J / \psi$. Unfortunately there is no data for $J / \psi \rightarrow \gamma f_{0}(500)$ because the background for the radiative decay of $J / \psi$ to scalars below $1 \mathrm{GeV}$. However we find a theoretical estimation for this process in which $\Gamma\left(J / \psi \rightarrow \gamma f_{0}(500)\right) \sim 50 \mathrm{eV}$ (Ref. ${ }^{7}$ ), Table 1 and comparison with experimental data (Refs. ${ }^{\dagger} 4,{ }^{* 1}$ and ${ }^{* 5,6}$ ).

Table 1. Results of the glueball decay into $\gamma \gamma$.

\begin{tabular}{cccc}
\hline Resonance & $J^{P C}$ & Theory $(\mathrm{keV})$ & Experimental $(\mathrm{keV})$ \\
\hline$f_{0}(500)$ & $0^{++}$ & 0.016 & $\dagger 1.7 \pm 0.4$ \\
$f_{0}(1500)$ & $0^{++}$ & $1.32 \times 10^{-3}$ & $* 0.095_{-0.017-0.060}^{+0.034+0.519}$ \\
$f_{0}(1710)$ & $0^{++}$ & 0.030 & $* 0.033_{-0.029}^{+0.975}$ \\
\hline
\end{tabular}

\section{Conclusions}

From our results, it appears that $2-\gamma$ decay widths of gluonium states tend, on the average, to be significantly smaller than the corresponding decay widths of most quarkonia decaying into two photons, as far as they have been measured. Results of others models have pointed out in the same direction.

We can see that the $f_{0}(1710)$ meson in Table 1 is a feasible glueball candidate because the theoretical result is in the experimental range.

The next step will be calculate the decay width to the others quantum numbers and make a mapping of the light meson sectors to the application of the model.

\section{Acknowledgments}

The authors thanks to M. V. T. Machado for useful discussions. This research was supported by CNPq and FAPERGS, Brazil. 


\section{References}

1. W. Ochs, J. Phys. G 40, 043001 (2013).

2. E. H. Kada, P. Kessler and J. Parisi, Phys. Rev. D 39, 2657 (1989).

3. L. Houra-Yaou, P. Kessler and J. Parisi, Phys. Rev. D 45, 794 (1992).

4. M. Hoferichter, D. R. Phillips and C. Schat, Eur. Phys. J. C 71, 1743 (2011).

5. S. Uehara et al. [Belle Collaboration], PTEP, 123C01 (2013).

6. M. Albaladejo and J. A. Oller, Phys. Rev. Lett. 101, 252002 (2008).

7. V. A. Navikov, M. A. Shifman, A. I. Vainshtein and V. I. Zakharov, Phys. Lett. B 165, 67 (1980). 